\title{
Automated Neuropsychological Assessment Metrics
}

National Cancer Institute

\section{Source}

National Cancer Institute. Automated Neuropsychological Assessment Metrics. NCI

Thesaurus. Code C49144.

A computerized library of tests designed for baseline screening and serial assessment of neurocognitive function. 